\title{
Correction of multiple nonlinear resonances in storage rings
}

\author{
R. Bartolini, ${ }^{1,2}$ I. P. S. Martin, ${ }^{1}$ and J. H. Rowland ${ }^{1}$ \\ ${ }^{1}$ Diamond Light Source, Oxfordshire, OX11 ODE, United Kingdom \\ ${ }^{2}$ John Adams Institute, University of Oxford, OX1 3RH, United Kingdom \\ P. Kuske \\ BESSY-II, 12489 Berlin, Germany \\ F. Schmidt \\ CERN, Geneva 23, Switzerland \\ (Received 17 July 2008; published 28 October 2008)
}

\begin{abstract}
The correct implementation of the nonlinear lattice model is crucial to achieving the design performance in storage rings. We describe here a method for the simultaneous correction of multiple nonlinear resonances based on local resonance measurements and numerical fits of the sextupole components. This method has been applied for the simultaneous correction of two sextupole resonances excited in the Diamond storage ring. The local correction of these resonances has been achieved with unprecedented precision. We also point out that this method has the potential to lead to an effective reconstruction of the local sextupole component errors around the whole ring circumference.
\end{abstract}

DOI: 10.1103/PhysRevSTAB.11.104002

PACS numbers: 05.45.- a, 29.20.db

\section{INTRODUCTION}

The performance of storage ring light sources or circular colliders depends crucially on the correct implementation of the nominal optics. Both the linear and the nonlinear optics are usually the result of a long and complicated process of optimization of the beam dynamics to achieve the small emittance with a long lifetime and good injection efficiency required in 3rd generation light sources or the large dynamic aperture and high luminosity required in circular colliders. While the correct implementation of the linear optics has been substantially eased by fitting algorithms such as LOCO [1], the calibration of the nonlinear model of the storage ring still lacks an analogously comprehensive approach. Important steps forward were made with the introduction of frequency map analysis [2] (FMA) which offers clear information on the detuning with amplitude and the resonance structures excited in the storage ring. A complementary technique based on the frequency analysis of the betatron motion has been proposed to measure the driving terms of the resonances $[3,4]$ that affect the beam motion and to establish their longitudinal dependence along the ring [5].

Within the latter theoretical formalism, we have developed a method for the calibration of the nonlinear model of the storage ring [6] which is based on a numerical fit of the sextupole components with the aim of reproducing the longitudinal dependence of the spectral lines and their corresponding resonance driving terms. The correct calibration of the linear optics is a prerequisite for the successful modeling of the nonlinearities in the storage ring and can be achieved with LOCO-type algorithms. This method, described in Sec. II, has been applied to both numerical tracking data and to machine turn-by-turn data taken at the Diamond storage ring. In Sec. III we report the result of the correction of the nonlinear lattice model. In Sec. IV we report the results of the application of this method to real machine data with the correction of two nonlinear resonances at the Diamond storage ring. Conclusions will be drawn in Sec. V.

\section{THE METHOD}

The frequency analysis of betatron motion can be used to extract information on the resonance driving terms that affect the nonlinear dynamics of the particle beam. It has been shown $[3,4]$ that each spectral line is proportional to a specific resonance driving term, at least in the first order of perturbation theory with the multipole components. We want to show that the measurement of the spectral lines can be used to correct the nonlinearities and to generate a model of the ring that reproduces the dynamics. This method is based on a least-squares fit of the lattice elements strength to minimize the difference between the spectral content measured from all beam position monitors (BPMs) in the machine and the ideal model. The spectral lines, whose amplitude and phase should be controlled, are selected according to which resonance appears to be most excited in the ring.

Correspondingly, the lattice parameters to be fitted should be selected among those that excite the selected resonance, e.g. sextupole components should be fitted for controlling spectral lines excited by third order resonances. The resonance driving terms vary along the ring and their value at each BPM can be obtained from the spectral decomposition of the betatron oscillation signal using a 
refined fast-Fourier-transform algorithm $[7,8]$ such as those developed in the NAFF [2,9] or SUSSIX [10] codes.

For a particular resonance that needs to be compensated for, since it significantly differs from the $s$-dependent pattern expected from the model, one can build a target vector $A_{\text {meas }}$ whose components are the amplitude and/or the phase of the spectral lines measured at each BPM. The detailed relationship between driving terms and spectral lines is reported in Refs. [3,4] and will not be repeated here. Indicating the generic resonance $m Q_{x}+n Q_{y}$ with $(m, n)$, we recall, as an example, that if the resonance $(3,0)$ is targeted, then the target vector to use is given by the amplitude and phase of the spectral line at a frequency $(-2,0)$ in the horizontal plane of motion, which is excited by the sextupoles in the ring

$$
\bar{A}_{\text {meas }}^{(-2,0)}=\left(A_{1}, \ldots, A_{\mathrm{Nbpm}} ; \phi_{1}, \ldots, \phi_{\mathrm{Nbpm}}\right),
$$

where Nbpm is the total number of BPMs in the ring. Each component of this vector will depend on the particular distribution of sextupoles in the ring, therefore we can write

$$
\bar{A}_{\text {meas }}^{(-2,0)}=\bar{A}_{\text {meas }}^{(-2,0)}\left(S_{1}, \ldots, S_{\text {Nsext }}\right),
$$

where $S_{k}$ is the strength of the $k$ th sextupole and Nsext is the total number of sextupoles.

This target vector is then compared with the same target vector computed from the ideal model $A_{\text {model }}$. The distance between these two vectors,

$$
\chi^{2}=\sum_{j=1}^{2 N b p m}\left[A_{\text {model }}^{(-2,0)}(j)-A_{\text {meas }}^{(-2,0)}(j)\right]^{2},
$$

gives a measure of the discrepancy between the real accelerator and the model. This quantity can be minimized by a least-squares minimization procedure that involves fitting the strength of the magnetic elements directly responsible for the excitation of that particular spectral line. Several algorithms can be used such as the singular value decomposition inversion of the Jacobian matrix which defines the dependence of the target vector $A$ on the fit parameter chosen:

$$
M_{j k}=\frac{\partial A_{j}}{\partial S_{k}} .
$$

We eventually opted for a Levenberg-Marquardt algorithm [11] built-in in the MATLAB optimization toolbox that proved quite effective and also allowed the introduction of constraints on the overall variation of the sextupole component. The Diamond storage ring has 168 BPMs and 168 sextupoles and one iteration for the fit takes typically 10 minutes for a target vector made of two resonances at all BPMs $(168 \times 2=336$ entries $)$ on an Intel core 2 processor.
Once the sextupole components have been fitted to reproduce the measured longitudinal dependence of the spectral lines, these components are used to correct the machine back towards the nominal model of the ring. The target vector can be built using the amplitude and/or the phase of several spectral lines according to the particular problem and the particular set of magnetic errors that we wish to control in the lattice.

Of course, the simple relationship between spectral lines and resonance driving terms is based on the perturbative analysis valid to the first order in the element strengths. This approach breaks down when higher orders become effective and the betatron motion is no longer quasiperiodic. The extension of these relations to higher order is discussed in Ref. [4].

\section{APPLICATION TO NUMERICAL TRACKING}

We investigated the effectiveness of the proposed method on the analysis of turn-by-turn data obtained from numerical tracking of a single particle in the nonlinear model of the Diamond storage ring. To simulate a realistic scenario, we introduced a series of random errors in the sextupole components and used the technique outlined in the previous section to reproduce the longitudinal pattern of the main spectral lines and therefore of the main resonance driving terms excited in the ring. We found that the method was capable of fully reconstructing the particular random sequence of errors that had been assigned to the sextupole component. In this way we prove that the method is capable of attacking the far more ambitious goal of individually identifying the local errors in the sextupole magnets.

The target vector was built using the amplitudes of the spectral lines of the resonances which were cleanly detectable by the analysis of the turn-by-turn data with SUSSIX. The applied kick excited betatron oscillations in both directions with the same amplitude. It was essential for the full reconstruction to use all the spectral lines that were identified at all BPMs by the SUSSIX program. In this case the target vector consisted of the amplitude of five spectral lines $(0,2),(-2,0),(-1,2),(-1,-2),(-3,0)$ detected in the horizontal plane of motion. The results of the analysis are shown in the Figs. 1 and 2 which show the comparison of the target vector before and after the fit. Figure 3 shows that all the individual random errors could be well identified.

We are quite aware of the fact that the phase information of the spectral line can be equally useful to extract relevant information on the nonlinear dynamics. Nevertheless, the mixing of amplitude and phase information in the target vector generates some difficulties related to the choice of the relative weighting of the two pieces of information. It is not yet fully clear how we may profit from the phase information. This deserves further analysis. 


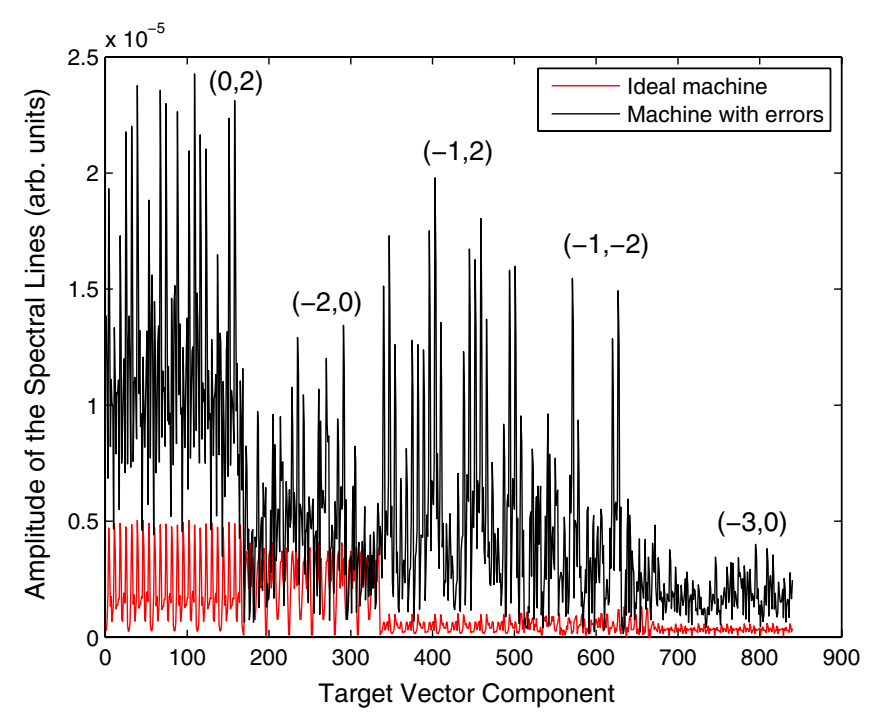

FIG. 1. (Color) Comparisons of the target vector built with the amplitude of five spectral lines $(0,2),(-2,0),(-1,2),(-1,-2)$, $(-3,0)$ in the horizontal plane for the nominal model for the Diamond bare lattice (red) and for the machine model with the assigned random errors in the sextupole (black).

The tracking simulations did not consider sources of errors such as BPM noise [12], BPM nonlinearities [13], decoherence [5] of the betatron oscillations, and radiation damping which naturally occur in a real storage ring.

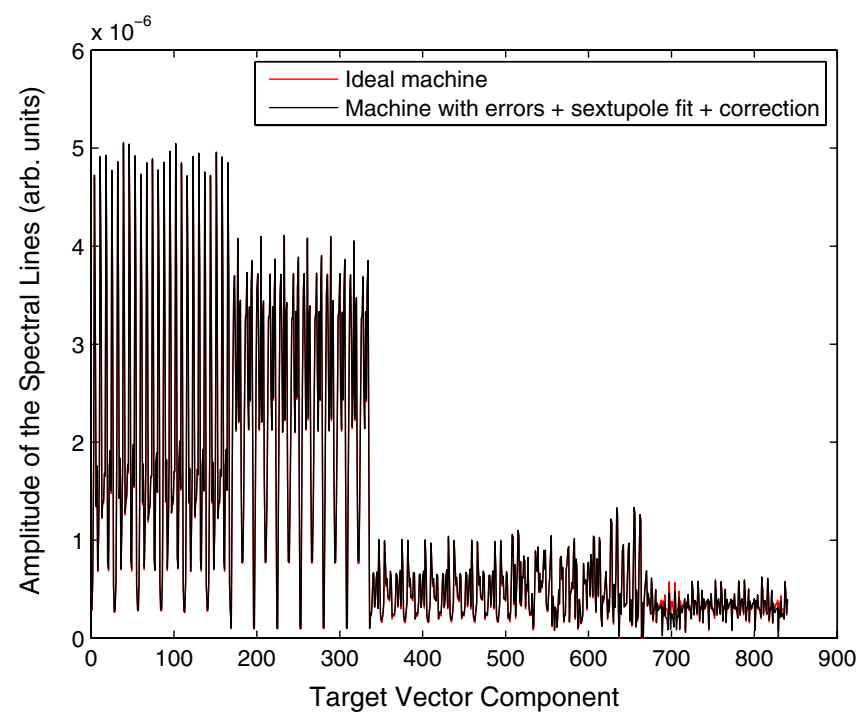

FIG. 2. (Color) Same comparison as in Fig. 1 after the sextupoles have been corrected with the fit procedure outlined in Sec. II. Notice that the vertical scale in this plot is smaller by roughly a factor of 4 compared to Fig. 1 . The nominal model for the Diamond bare lattice is in red and the machine model with random errors in the sextupole component and their correction in black. Notice also that the red line is barely visible underneath the black line. This demonstrates how well this correction technique may work.

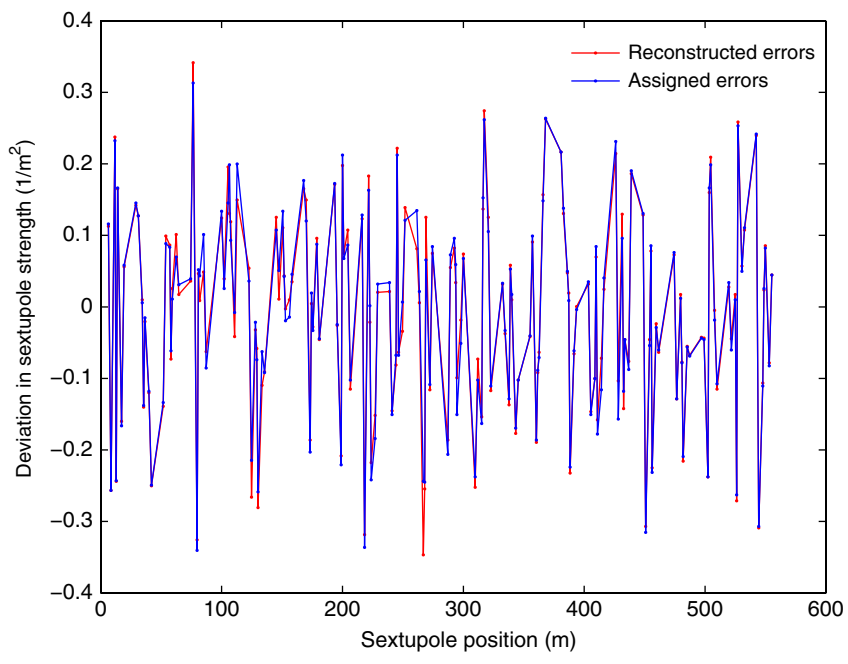

FIG. 3. (Color) Assigned sextupole components (blue) and their reconstructed values with the numerical fit (red).

Therefore the full reconstruction of the nonlinear model of the real accelerator can only be achieved within the limits that these errors impose.

\section{EXPERIMENTAL RESULTS AT DIAMOND}

A campaign of measurements was started with the aim of measuring the local longitudinal dependence of the resonant driving terms, comparing them with the nonlinear ring model and correcting the deviations by means of the method outlined in the previous sections. The Diamond storage ring is equipped with two independent pinger magnets that can excite betatron oscillations to large amplitudes, it has turn-by-turn capabilities at all BPMs, and has independently powered sextupole magnets. The experi-

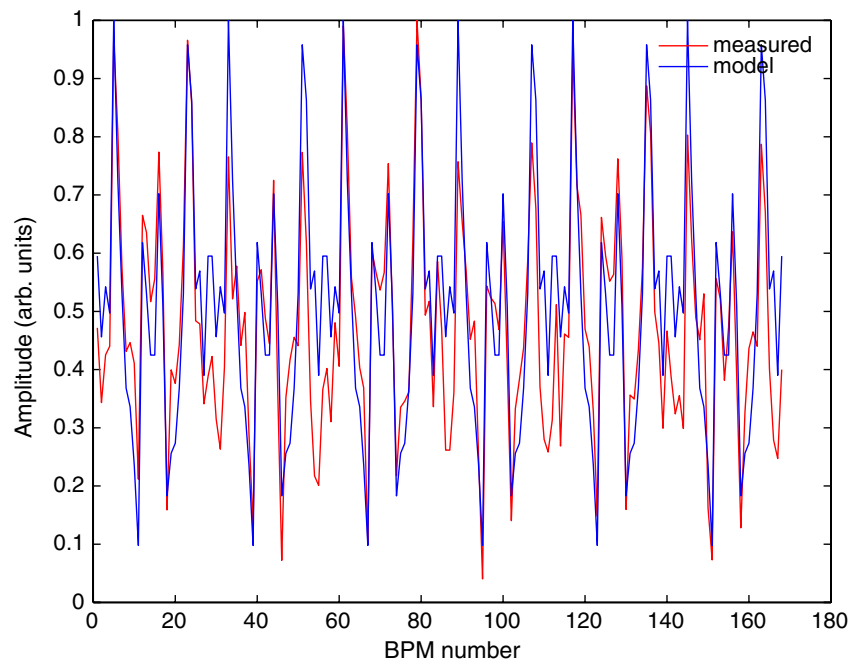

FIG. 4. (Color) Comparison of the amplitude of the $(-1,1)$ line in the vertical plane. The spectral line from the model is blue while the measured one is in red. 


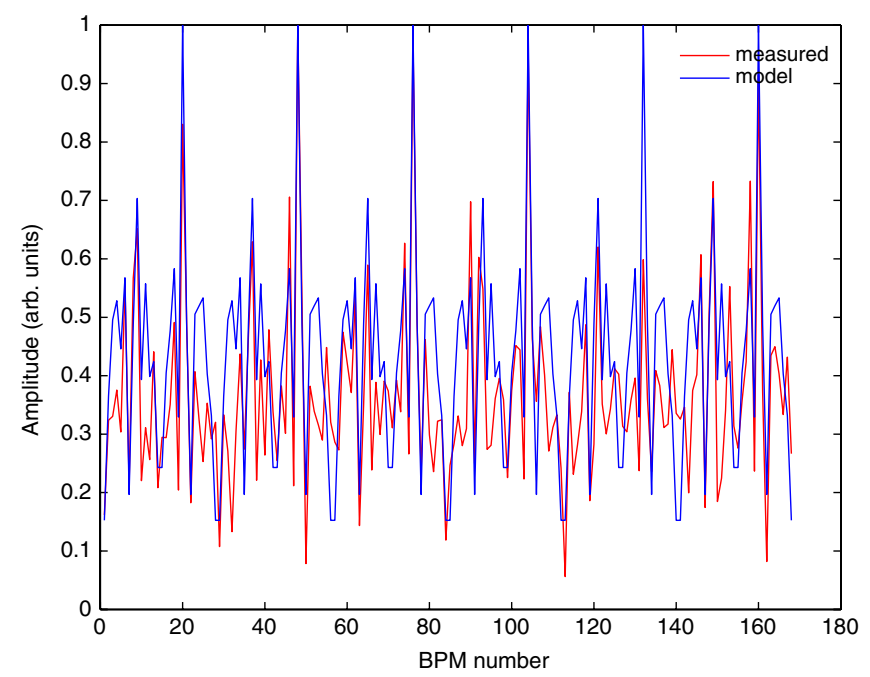

FIG. 5. (Color) Comparison of the amplitude of the $(-2,0)$ line in the horizontal plane. The spectral line from the model is blue while the measured one is in red.

ments were always performed after an initial correction of the linear optics with LOCO [1]. A residual $\beta$ beating of less than $1 \%$ peak to peak and a coupling correction better than $0.2 \%$ were routinely achieved. We applied the method described in the previous sections to correct the longitudinal dependence of the spectral lines excited by nonlinear resonances. The pinger magnets were used to excite betatron oscillations simultaneously in both planes, although aperture limitations did not allow diagonal kicks to large amplitudes. The turn-by-turn data of these experiments were generated with horizontal kicks 4 times larger than the vertical ones. We could detect the spectral lines associated to the resonances $(1,-2)$ as seen in the vertical plane (Fig. 4) and the resonance $(3,0)$ as seen in the horizontal plane (Fig. 5). The comparison with the model is already good without any correction of the sextupole components, nevertheless some discrepancies appear and these were the object of our investigation.

We have first tried the correction of a single nonlinear resonance, in the hope that the other resonances would also be reduced. The fit of the sextupole components converged to a new setting of the sextupoles which reproduced very well the spectral lines along the ring at all BPMs as shown in Fig. 6. Nevertheless we observed that other spectral lines, neglected in the minimization target, were indeed poorly controlled and actually increased as a result of the fit: Fig. 7 reports the difference between the measured and model spectral line $(1,-1)$ controlled by the fit and the $(-2,0)$ line excluded from the fitting procedure, which indeed appears to grow after two iterations. The contribution to the $\chi^{2}$ of the $(-1,1)$ line is reduced by almost a factor of 4 while the contribution to the $\chi^{2}$ of the $(-2,0)$ line is increased by $25 \%$. The sextupole variation required
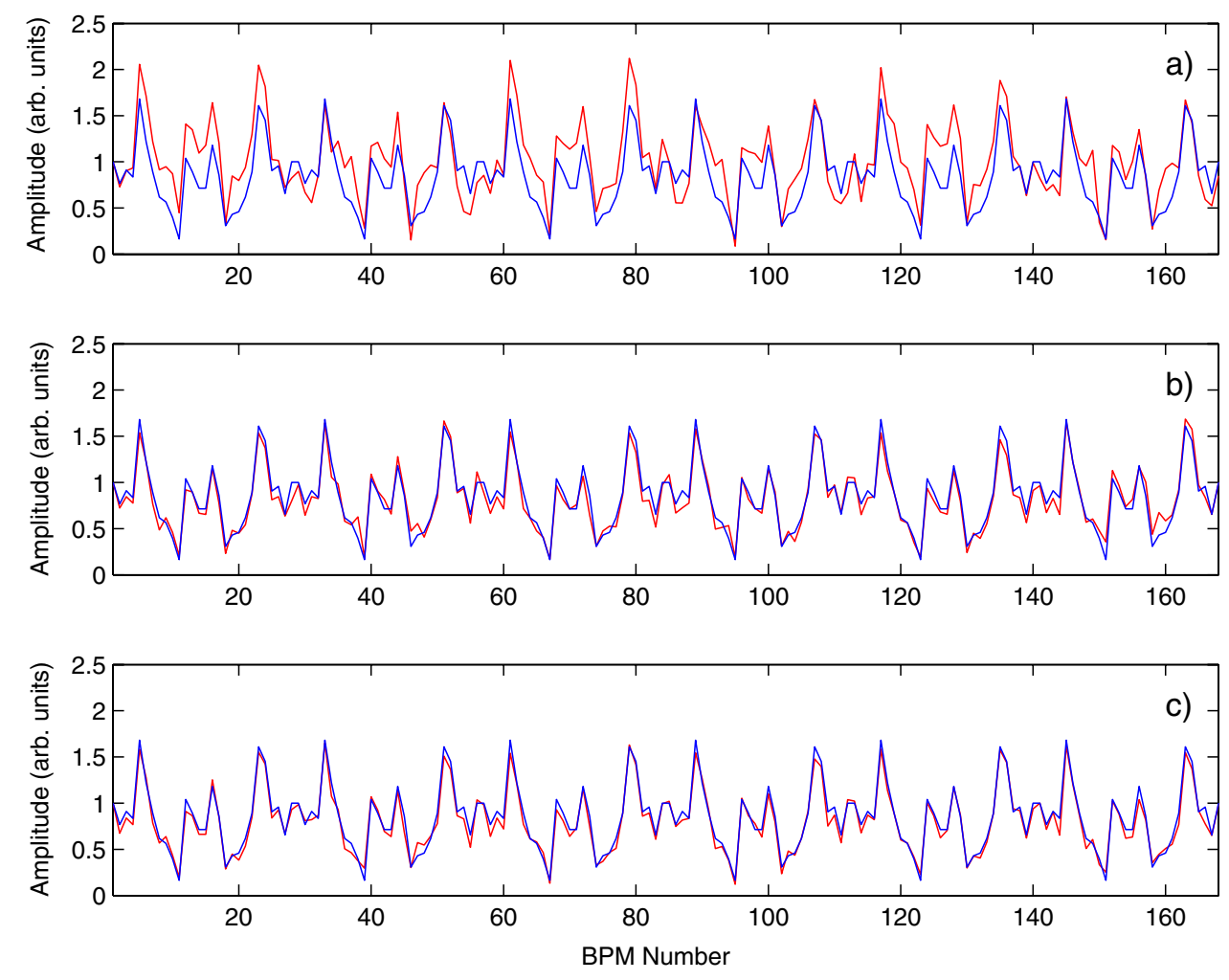

FIG. 6. (Color) Correction of a single resonance by fitting the $(-1,1)$ line in the vertical plane: before correction (a); after one iteration (b); after two iterations (c). The spectral line from the model is blue while the measured one is in red. 

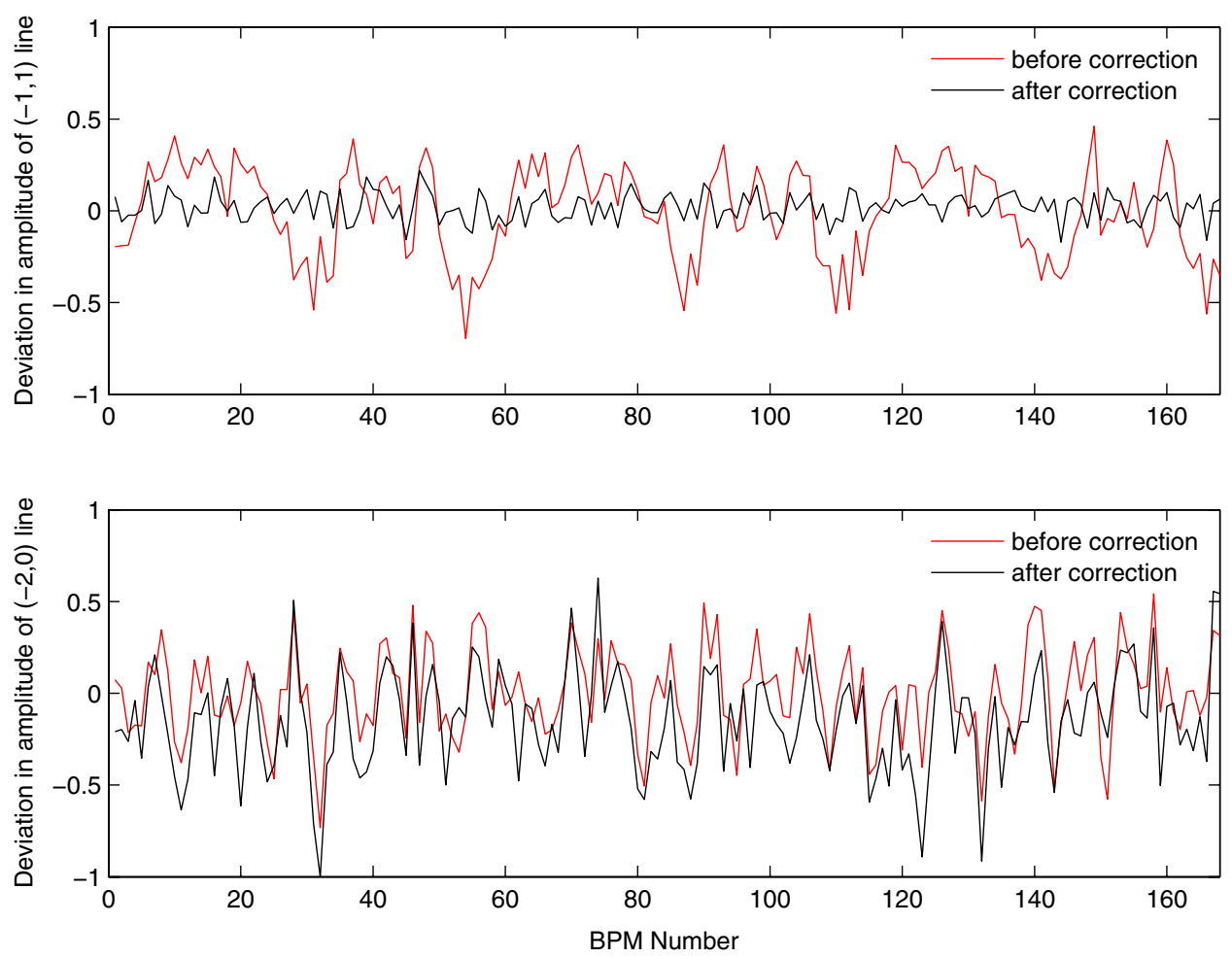

FIG. 7. (Color) Difference of the two spectral lines $(-1,1)$ and $(-2,0)$ at the beginning and at the end of the fit procedure (targeting only one resonance).
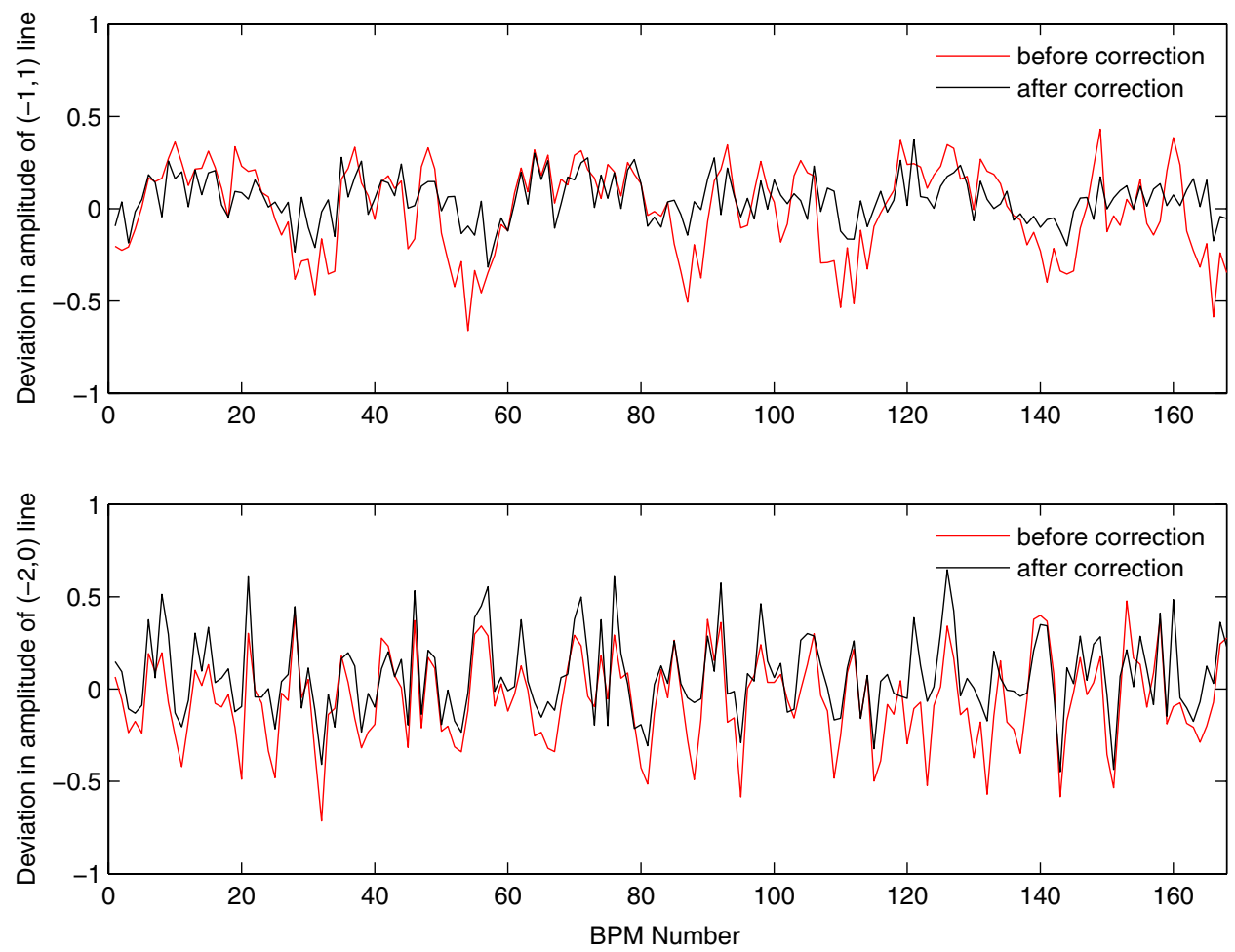

FIG. 8. (Color) Simultaneous reduction of the $(-1,1)$ line in the vertical plane and of the $(-2,0)$ line in the horizontal plane after the sextupole component variation determined by the fit procedure. 


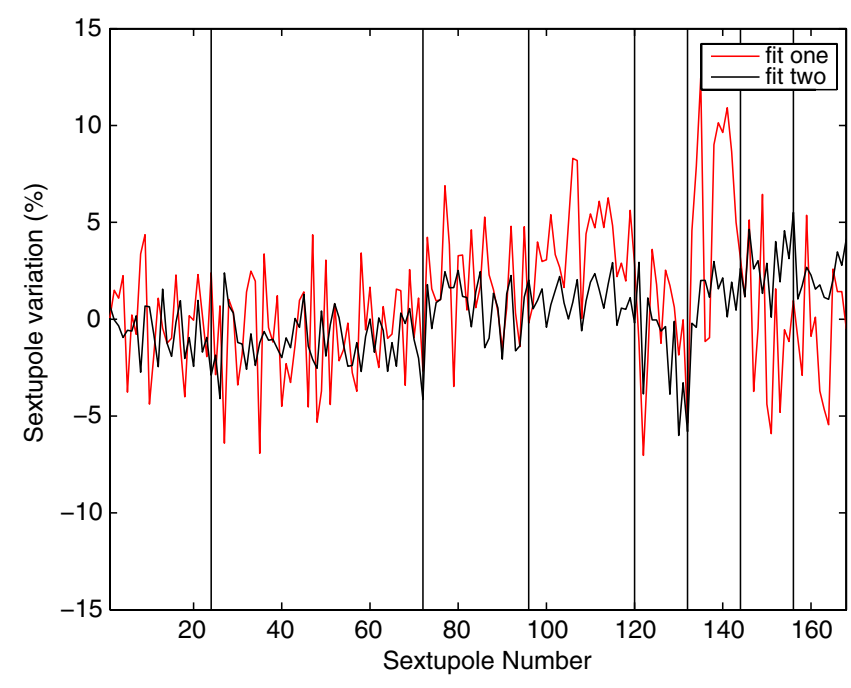

FIG. 9. (Color) Sextupole component variation introduced by the fit in Fig. 7 (red) and in Fig. 8 (black).

by the fit reached $10 \%$ which is deemed to be incompatible with magnetic measurements results.

We therefore included a second resonance line to the target. In this case, the sextupole fit converged with substantially reduced variations of the sextupole strengths along the ring as shown in Fig. 8. The contribution to the $\chi^{2}$ of the $(-1,1)$ line is reduced by almost a factor of 2 and the contribution to the $\chi^{2}$ of the $(-2,0)$ line was also reduced by $20 \%$. The resulting sextupole variation is reported in Fig. 9, and shows that now the sextupole variation required is always less than 5\% peak to peak. Applying these sextupole strength corrections to the real machine resulted in an increase in lifetime by $10 \%$. This is a clear demonstration, in storage ring light sources, that a deterministic improvement of nonlinear beam dynamics leads to an improvement of the performance of the storage ring.

\section{CONCLUSION AND ACKNOWLEDGMENTS}

We have presented a new technique for the correction of the nonlinear resonances in a storage ring and proven its effectiveness both in numerical tracking, where this technique can reproduce the nonlinear model of the ring, and in real experimental data where a global improvement of the nonlinear dynamics was achieved resulting in an increase of the lifetime of the Diamond storage ring. The method proposed provides a deterministic strategy for the simultaneous correction of multipole resonances, taking fully into account their longitudinal dependence and it goes beyond the compensation of nonlinear resonances performed so far in synchrotrons [14]. The method requires independently powered sextupole magnets. The benefits of the correction of the leading terms of the nonlinear resonances with sextupole powered in families will be the object of forthcoming investigations.

This method provides useful information on the nonlinear part of the ring model, complementary to those obtained by the FMA, by looking at the whole spectral content of the betatron oscillations computed at all BPMs, rather than the tune dependence with amplitude computed at one BPM. Furthermore, we would like to stress that the applicability of these techniques is not restricted to synchrotron light sources but is equally valid for colliders where typically higher order multipoles typically dominate the nonlinear beam dynamics.

\section{ACKNOWLEDGMENTS}

We would like to thank G. Rehm and the Diamond Diagnostics Group for the continuous support with the setup and operations of the pinger magnets and the BPM system.

[1] J. Safranek, Nucl. Instrum. Methods Phys. Res., Sect. A 388, 27 (1997).

[2] J. Laskar, Physica D (Amsterdam) 67, 257 (1993).

[3] J. Bengtsson, CERN Report No. 88-05, 1988.

[4] R. Bartolini and F. Schmidt, Part. Accel. 59, 93 (1998).

[5] R. Tomas Garcia, Ph.D. thesis, University of Valencia [Report No. CERN-THESIS- 2003-010, 2003 (unpublished)].

[6] R. Bartolini and F. Schmidt, Proceedings of the 2005 Particle Accelerator Conference Knoxville, Tennessee, USA, edited by C. Horak, p. 1452.

[7] E. Asseo, Report No. CERN/PS/LEA Note 87-1, 1987.

[8] R. Bartolini et al., Part. Accel. 52, 147 (1996).

[9] D. Robin et al., Phys. Rev. Lett. 85, 558 (2000).

[10] R. Bartolini and F. Schmidt, Report No. CERN-SL 98-017 (AP), 1997.

[11] W. H. Press et al., Numerical Recipes in C (Cambridge University Press, Cambridge, England, 1992), Chap. 15.5.

[12] R. Bartolini et al., Part. Accel. 55, 247 (1996).

[13] R. W. Helms et al., Phys. Rev. ST Accel. Beams 8, 062802 (2005).

[14] M. Benedikt et al., Phys. Rev. ST Accel. Beams 10, 034002 (2007). 